\title{
$O$-GIcNAc modification influences endometrial receptivity by promoting endometrial cell proliferation, migration and invasion
}

\author{
XIAO HAN $^{1 *}$, XIAOXUE LI $^{1 *}$, HUAN LIU $^{1}$, HONGSHUO ZHANG $^{1}$, AOYING LI $^{2}$, \\ MING DONG ${ }^{1}$, YUEFEI XU ${ }^{1}$, BIN YAN $^{3}$, LINLIN SUI ${ }^{1}$ and YING KONG ${ }^{1}$ \\ ${ }^{1}$ Core Laboratory Glycobiol and Glycogen, College of Basic Medical Sciences, \\ ${ }^{2}$ Clinical Medicine of 2016, Dalian Medical University; ${ }^{3}$ Department of Gynecology, \\ The First Affiliated Hospital of Dalian Medical University, Dalian, Liaoning 116044, P.R. China
}

Received March 28, 2019; Accepted July 15, 2019

DOI: $10.3892 / o r .2019 .7317$

\begin{abstract}
O$-linked $\beta$-N-acetylglucosamine $(O$-GlcNAc) modification is a dynamic post-translational modification process that is involved in many crucial biological processes, including cell cycle regulation, nutrient metabolism and extracellular signaling. This dynamic modification is dependent on the ambient glucose concentration and is catalyzed and removed by $O$-GlcNAc transferase (OGT) and $O$-GlcNAcase (OGA), respectively. The present study aimed to determine the role of $O$-GlcNAcylation during embryo implantation by inhibiting or enhancing its function and expression. The results revealed that the expression of $O$-GlcNAc-modified proteins in the human secretory endometrium was higher than that of the endometrium during the proliferative phase, as determined via western blotting and immunohistochemistry. Additionally, the level of endometrial $O$-GlcNAc modification increased gradually from the pre-receptive to the receptive phase, which was then decreased during the non-receptive phase. In endometrial cells, RNA interference was utilized to reduce the expression of two key $O$-GlcNAc synthesis and decomposition enzymes, OGT and OGA, to indirectly increase or decrease levels of $O$-GlcNAc modification. The results revealed that increasing the level of $O$-GlcNAc modification enhanced cellular proliferation, migration, invasion and adhesion, thereby promoting embryo implantation. It is hypothesized that $O$-GlcNAc modification serves an important role in the regulation of endometrial receptivity and embryo implantation. The results of the present study may have important implications for
\end{abstract}

Correspondence to: Dr Linlin Sui or Dr Ying Kong, Core Laboratory Glycobiol and Glycogen, College of Basic Medical Sciences, Dalian Medical University, 9 West Section, Lvshun South Road, Dalian, Liaoning 116044, P.R. China

E-mail: linlin19810622@sina.com

E-mail: kongying@ dmu.edu.cn

${ }^{*}$ Contributed equally

Key words: $O$-linked $\beta$-N-acetylglucosamine, $O$-GlcNAc transferase, $O$-GlcNAcase, endometrial receptivity, endometrial cells the understanding of female fertility and may help improve infertility treatments.

\section{Introduction}

$O$-GlcNAcylation is a reversible post-translational modification whereby a single $N$-acetylglucosamine (GlcNAc) molecule is added to the hydroxyl group of Ser/Thr protein residues. This reaction is catalyzed by the enzyme, $O$-linked- $N$-acetylglucosamine transferase (OGT) and the protein sugar moiety is removed using $O$-GlcNAcase enzymes (OGA, NCOAT or MGEA5) $(1,2)$.

Endometrial receptivity refers to a limited period of time during which the endometrium is conducive to blastocyst growth, attachment and the subsequent events of implantation $(3,4)$. The sensitivity of the endometrium to the embryo is classified as the pre-receptive, receptive and non-receptive phases (5). During the pre-receptive phase, the uterus cannot initiate embryo implantation. However the uterine environment does not pose a specific threat to the survival of the embryo. During the non-receptive phase, the uterine environment is not conducive to the survival of the embryo.

The female menstrual cycle lasts for an average of 28-30 days and is divided into the proliferative (follicular) and secretory (luteal) phases. The uterus then becomes receptive to blastocyst attachment in the mid-luteal phase for 4 days (7-10 days after ovulation) (6). Normal cycle duration is a key determinant of whether an embryo can be implanted smoothly. Embryo implantation is the main rate-limiting step in pregnancy and endometrial receptivity is closely associated with embryo implantation. Endometrial receptivity can also affect implantation and clinical pregnancy rates. The establishment of endometrial receptivity is therefore important to ensure the successful development of the fetus and placenta.

Previous studies have focused on the role of protein glycosylation modification in implantation-associated events, including embryonic development and maternal-fetal interface recognition. The results revealed that $\mathrm{Le}^{\mathrm{Y}}$ glycan, fucosyltransferase and $\beta 1,4$-galactosyltransferase regulate embryo implantation, indicating that glycosylation modification exerts an important effect on implantation $(7,8)$. 
At present, our understanding of $O$-linked $\beta-N$-acetylglucosamine $(O$-GlcNAc) modifications is mainly in the context of tumor occurrence and metastasis (9), yet the behavior of invasive cancer cells is similar to that of invasive placental cells (10). Protein glycosylation serves an important role in embryonic development, but the role of $O$-GlcNAcylation in the regulation of implantation is yet to be fully elucidated. The present study utilized RL95-2 and HEC-1A endometrial carcinoma cell lines to simulate high-receptive and low-receptive endometrial states, respectively. A chorionic tumor cell line, JAR, was used to simulate invasive embryos (11). Using this model, the present study aimed to determine the role of $O$-GlcNAcylation during the process of embryo implantation. The results of the present study may therefore have important significance for the improvement and treatment of female fertility. The results may also provide a theoretical basis for the development of clinical reproductive regulation, pregnancy aid and anti-implantation technology.

\section{Materials and methods}

Patients and samples. Endometrial tissue $(\mathrm{n}=36$; median age, 35 years; age range, 25-45 years; 18 cases in each of the proliferative and secretory stages) were obtained from The First Affiliated Hospital of Dalian Medical University under the Human Research Agreement approved by the Ethics Committee of Dalian Medical University. Written informed consent was acquired from each patient prior to tissue collection. Endometrial specimens were collected, embedded in paraffin wax and screened via histopathology.

The patients included in the present study were diagnosed with gynecological benign uterine leiomyoma and were undergoing hysterectomy. They exhibited normal fertility and a regular menstrual cycle. All patients had not received any hormonal drugs for a minimum of 3 months. Patients with diabetes, hyperthyroidism, hypothyroidism, ovarian neoplasms and other complications were excluded.

Cell lines and cell culture. RL95-2 and HEC-1A endometrial carcinoma cell lines and the chorionic tumor cell line, JAR, were purchased from the American Type Culture Collection (ATCC). The JAR cell line was established from a trophoblastic tumor of the placenta, but the cell line was derived from a male fetus and as such should not affect the results of the present study. RL95-2 cells were cultured in DMEM/F12 media (Gibco; Thermo Fisher Scientific, Inc.) supplemented with $0.1 \%$ insulin, $10 \%$ fetal bovine serum (FBS) (ScienCell Research Laboratories, Inc.) and $1 \%$ penicillin/streptomycin (Invitrogen; Thermo Fisher Scientific, Inc.). JAR cells were cultured in DMEM/F12 media (Gibco; Thermo Fisher Scientific, Inc.) supplemented with $10 \%$ FBS and $1 \%$ penicillin/streptomycin (Invitrogen; Thermo Fisher Scientific, Inc.). HEC-1A cells were maintained in McCoy's 5A media (Gibco; Thermo Fisher Scientific, Inc.), supplemented with $10 \%$ FBS and $1 \%$ penicillin/streptomycin (Invitrogen; Thermo Fisher Scientific, Inc.). All cell lines were maintained at $37^{\circ} \mathrm{C}$ in a humidified atmosphere containing $5 \% \mathrm{CO}_{2}$. Growth media were changed every 2-3 days.

RNA interference. Small interfering RNA (siRNA) duplexes targeting the OGT and OGA genes, as well as an siRNA negative control, were synthesized by GenePharma Co., Ltd. The following siRNA sequences were utilized: OGT sense, 5'-GCA GUAACACAGCUCUUAATT-3' and antisense, 5'-UUA AGAGCUGUGUUACUGCTT-3'; OGA sense, 5'-GGGAUA UCAAGAGUAUAAUTT-3' and antisense, 5'-AUUAUACUC UUGAUAUCCCTT-3'; negative control sense, 5'-UUCUCC GAACGUGUCACGUTT-3' and antisense, 5'-ACGUGACAC GUUCGGAGAATT-3'. siRNA transfection was performed in HEC-1A and RL95-2 cells. Cells were transfected with 100 pmol siRNA using Lipofectamine 2000 (Invitrogen; Thermo Fisher Scientific, Inc.) in accordance with the manufacturer's protocol.

Immunohistochemistry. Human endometrial tissues were sectioned $(4 \mu \mathrm{m})$ and dried at $56^{\circ} \mathrm{C}$ for $60 \mathrm{~min}$. After the de-paraffinization and re-hydration of tissue sections, antigen retrieval was performed at $100^{\circ} \mathrm{C}$ for $20 \mathrm{~min}$ in citrate buffer. Samples were then treated with $3 \%$ peroxide for $15 \mathrm{~min}$ at room temperature in the dark to quench endogenous peroxidase activity. Sections were washed in PBS and blocked for $15 \mathrm{~min}$ with $5 \%$ goat serum at $37^{\circ} \mathrm{C}$ to prevent non-specific binding. Following incubation with an $O$-GlcNAc primary antibody (Abcam; cat. no. ab2739; dilution 1:50) overnight at $4^{\circ} \mathrm{C}$, the sections were washed several times. Biotinylated secondary antibodies (OriGene Technologies, Inc.; cat. no. sp9000; dilution 1:1) were then added to the tissue sections and incubated at $37^{\circ} \mathrm{C}$ for $30 \mathrm{~min}$. After washing, the sections were incubated with streptavidin-horseradish peroxidase (OriGene Technologies, Inc.) at $37^{\circ} \mathrm{C}$ for $30 \mathrm{~min}$. Sections were then allowed to react with diaminobenzidine (DAB)-peroxidase substrate (OriGene Technologies, Inc.), counterstained with hematoxylin for $30 \mathrm{sec}$, dehydrated and mounted in distrene dibutylypthalate xylene. Photomicrographs were captured using an Olympus TH4-200 microscope (magnifications, $\mathrm{x} 10$ and $\mathrm{x} 40$ ). The collected images were analyzed and processed using an Image-Pro Plus 6.0 (Media Cybernetics) image analysis system. The mean optical density of positively stained areas of endometrial stromal cells, glandular epithelial cells and luminal epithelial cells was measured. PBS replaced the primary antibody for negative controls.

Reverse transcription-quantitative PCR (RT-qPCR). Total RNA was isolated from cells using TRIzol reagent (Takara Biotechnology Co., Ltd.), according to the manufacturer's protocol. RNA samples were quantified via spectrophotometry at 260 and $280 \mathrm{~nm}$, and exhibited a 260/280 $\mathrm{nm}$ ratio ranging from 1.8-2.0, which indicated a high quality of extracted RNA. RNA $(1.0 \mu \mathrm{g})$ was subsequently reverse transcribed into cDNA using the All-in-One First-Strand cDNA Synthesis SuperMix for qPCR (One-Step gDNA Removal; Beijing TransGen Biotech Co., Ltd.) following the manufacturer's protocol. qPCR analysis was performed in a $10 \mu \mathrm{l}$ reaction system containing: $5 \mu 12 \mathrm{X}$ SYBR Premix Ex Taq (Beijing TransGen Biotech Co., Ltd.; AT341), $0.2 \mu 1$ of each forward and reverse primer $(10 \mu \mathrm{M}), 0.2 \mu 1$ ROX Reference Dye I (50X)

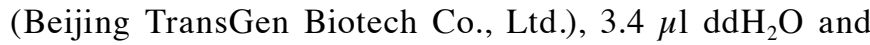
$1.0 \mu 1 \mathrm{cDNA}$. The Applied Biosystems ABI Step One Plus real-time PCR system (Thermo Fisher Scientific, Inc.) was utilized. Relative gene expression was determined using the $2^{-\Delta \Delta \mathrm{Cq}}$ method $(12,13)$ with GAPDH as the reference gene. All RT-qPCR reactions were performed in triplicate using the 
following primer sequences designed with oligo7: GAPDH (XM_001256799.2) forward, 5'-GTGAAGGTCGGAGTC AACG-3' and reverse 5'-TGAGGTCAATGAAGGGGTC-3'; OGT (XM_017029908.1) forward, 5'-CGGGAATCACCC TACTTCACACC-3' and reverse, 5'-CCGCCATCACCTTCA CTCGAAA-3'; OGA (XM_017015586.1) forward, 5'-TCC CCAGAGATGTCCATGCAAG-3' and reverse, 5'-TCCTTT GGGTCCATGCTCGTA-3'.

Western blot analysis. Proteins from endometrial tissues or cells were extracted using lysis buffer (Nanjing KeyGen Biotech Co., Ltd.) in accordance with the manufacturer's protocol. The concentration of protein extracts was determined using a BCA Protein Quantitative kit (TransGen Biotech Co., Ltd.). Protein $(40 \mu \mathrm{g})$ was denatured in 6X Protein Loading Buffer for $7 \mathrm{~min}$ at $100^{\circ} \mathrm{C}$. Proteins were then separated via $8 \%$ SDS-PAGE and transferred to nitrocellulose membranes (EMD Millipore) for $90 \mathrm{~min}$ at $4^{\circ} \mathrm{C}$. Membranes were blocked with $5 \%$ non-fat dried milk in Tris-buffered saline $(10 \mathrm{mmol} / \mathrm{l}$ Tris, $\mathrm{pH} 7.5$ and $0.14 \mathrm{~mol} / \mathrm{l} \mathrm{NaCl}$ ) with $0.1 \%$ Tween-20 (TBST) for $2 \mathrm{~h}$ at room temperature, followed by incubation with the following primary antibodies overnight at $4^{\circ} \mathrm{C}$ : Anti- $O$-GlcNAc (Abcam; cat. no. ab2739; dilution 1:1,000), anti-OGT (ProteinTech Group, Inc.; cat. no. 11576-2-AP; dilution 1:1,000), anti-OGA (ProteinTech Group, Inc.; cat. no. 14711-1-AP; dilution 1:1,000), anti-cyclin D1 (ProteinTech Group, Inc.; cat. no. 60186-1-Ig; dilution 1:2,000), anti-B-cell lymphoma-xL (Bcl-xl; CLOUD-CLONE Corp.; cat. no. PAE582Hu01; dilution 1:700), anti-matrix metalloproteinase-9 (MMP9; Abcam; cat. no. ab38898, dilution 1:1,000), MMP2 (Bioworld Technology, Inc.; cat. no. bs1236; dilution 1:700), anti-Snail (ProteinTech Group, Inc.; cat. no. 26183-1-AP; dilution 1:900), anti-E-cadherin (ProteinTech Group, Inc.; cat. no. 20874-1-AP, dilution 1:700) and anti- $\beta$-actin (ProteinTech Group, Inc.; cat. no. 20536-1-AP; dilution 1:2,000). After being washed three times with TBST, the membranes were probed with horseradish-peroxidase-conjugated anti-mouse or anti-rabbit IgG secondary antibodies (ProteinTech Group, Inc.; cat. nos. SA00001-1 and SA00001-2; dilution 1:3,000) at room temperature for $1 \mathrm{~h}$ and subsequently washed further with TBST. Immunoreactive bands were visualized using an enhanced chemiluminescence detection system, according to the manufacturer's protocol. The entire experiment was repeated three times. Image Lab software (Bio-Rad Laboratories, Inc. Ver: 4.0 49710) was used for the detection and analysis of protein bands. Protein expression was quantified via densitometric analysis and expression was normalized to that of $\beta$-actin.

Cell adhesion assay. RL95-2 and HEC-1A cells $\left(5 \times 10^{6}\right)$ were transfected in 6-well plates for $12 \mathrm{~h}$. Together with the cells of the untreated group, the transfected cells were digested with trypsin and cultured in a 24-well plate for $12 \mathrm{~h}$ to ensure that cells covered the entire surface area. CellTraceCFSE (Invitrogen; Thermo Fisher Scientific, Inc.; cat. no. C34554; $5 \mu \mathrm{g}$ ) was dissolved in $10 \mu \mathrm{l}$ of DMSO and diluted with media at a ratio of 1:2,000. JAR cells were pretreated with diluted carboxyfluorescein succinimidyl ester for $30 \mathrm{~min}$. The JAR cell suspension was subsequently added to 24 -well plates at a $1: 1$ ratio and incubated for $1 \mathrm{~h}$ at $37^{\circ} \mathrm{C}$ in $5 \% \mathrm{CO}_{2}$ and a $90 \%$ humidity. Fluorescent tags were added and a fluorescence microscope was used for imaging (x10) and cell counting. The number of fluorescently labeled cells was counted from five randomly selected fields under a fluorescence microscope and the results of at least three independent experiments.

Cell counting kit-8 (CCK-8) assay. HEC-1A or RL95-2 cells from control and siRNA groups were trypsinized and seeded into 96-well culture plates at a density of 5,000 cells/well using the prescribed media supplemented with 10\% FBS. Each group was provided with three multiple holes. The proliferation assay was performed using a CCK-8 kit (Dojindo Molecular Technologies, Inc.) in accordance with the manufacturer's protocol. Following culture for 24, 48 and $72 \mathrm{~h}, 10 \mu \mathrm{l}$ of CCK-8 solution was added to each well and incubated for $2 \mathrm{~h}$ at $37^{\circ} \mathrm{C}$. Optical density (OD) values of absorbance for each well were measured at $450 \mathrm{~nm}$ using Fluoroskan Ascent FL (Thermo Fisher Scientific, Inc.). Representative results from three independent experiments were expressed as the mean \pm SEM for each condition.

Wound healing assay. After pretreatment (O-GlcNAc knockdown or overexpression), HEC-1A or RL95-2 cells $\left(5 \times 10^{6}\right)$ were seeded in 6-well plates for $24 \mathrm{~h}$. The resulting cell monolayer was then wounded by removing a 500 - to $700-\mu \mathrm{m}$ wide strip with a standard $200-\mu 1$ pipette tip, after which samples were washed twice with PBS to remove floating cells. An optical Olympus IX71 microscope (Olympus Corp.; magnification, x10) was used to image specific sites on the first day post-wound. Cells were cultured in media supplemented with $10 \% \mathrm{FBS}$ in $5 \% \mathrm{CO}_{2}$ at $37^{\circ} \mathrm{C}$ for a further 24 or $48 \mathrm{~h}$ and imaged at 2 or 3 days, respectively. Wound healing was quantified by measuring the migratory distance of cells.

Transwell invasion assay. The invasion of cells was assessed using a 24-well Transwell Permeable Support with $8-\mu$ m pores (Corning, Inc.) according to the manufacturer's protocol. The inner compartments of the Transwell inserts were pre-coated with $50 \mu \mathrm{l}$ of $1 \mathrm{mg} / \mathrm{ml}$ Matrigel matrix (BD Biosciences) at $37^{\circ} \mathrm{C}$ for $1 \mathrm{~h}$ to solidify samples. RL95-2 and HEC-1A cells $\left(1 \times 10^{5}\right.$ cells $\left./ \mathrm{ml}\right)$ in serum-free media were seeded into the upper chamber of the Matrigel-coated filter. Media containing $10 \%$ FBS $(500 \mu 1)$ was subsequently added to the lower chamber as a chemoattractant. After incubation for $24 \mathrm{~h}$ at $37^{\circ} \mathrm{C}$ in $5 \% \mathrm{CO}_{2}$, non-invading cells were removed from the upper surface of the filter membrane. Invading cells that remained on the bottom surface of the filter membrane were fixed in methanol and stained with crystal violet for $15 \mathrm{~min}$ at room temperature. The number of invaded cells on each membrane was counted from five randomly selected fields under a light microscope (Olympus Corp., magnification, x20).

Statistical analyses. All data of the present study are the results of at least three independent experiments. Data are normally presented as the mean \pm SEM. A Student's t-test was used to compare the statistical significance between two groups. SPSS version 16.0 for Windows (SPSS, Inc., Chicago, IL, USA) was used for statistical analyses. $\mathrm{P}<0.05$ was considered to indicate a statistically significant difference. P-values were indicated in the figures and legends as follows: ${ }^{* * *} \mathrm{P}<0.001,{ }^{* *} \mathrm{P}<0.01$ and ${ }^{*} \mathrm{P}<0.05$. 

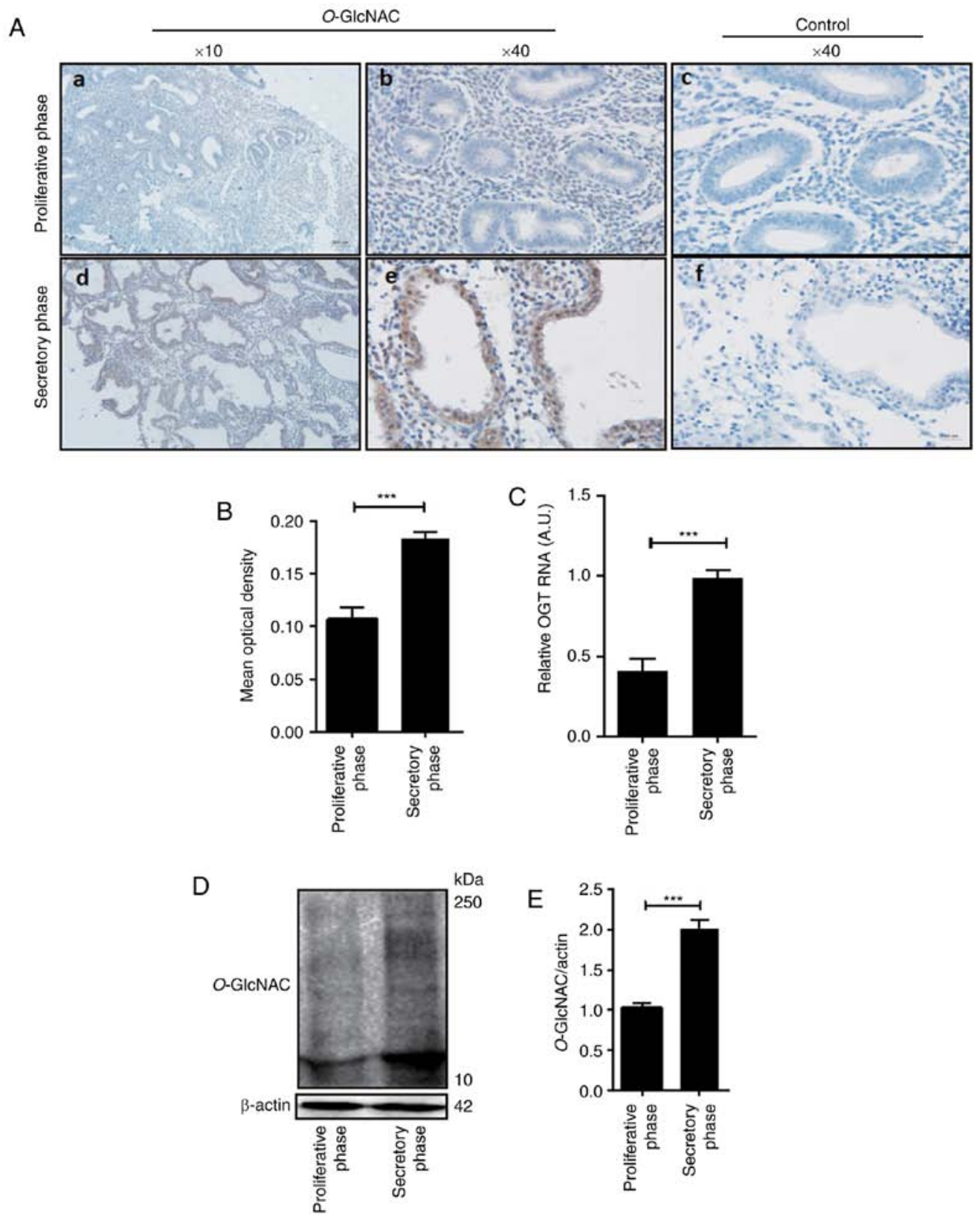

Figure 1. Expression of $O$-GlcNAc-modified proteins in human endometrial tissue. (A) (a and b) Micrographs of immunostaining for $O$-GlcNAc in endometrial tissue during the proliferative phase and in ( $\mathrm{d}$ and e) endometrial tissues during the secretory phase (magnification, $\mathrm{x} 10$ and $\mathrm{x} 40$ ). (c and $\mathrm{f}$ ) Negative control (magnification, x40) incubated with PBS. (B) The mean optical density of areas with positive immunostaining from endometrial tissue. (C) Analysis of OGT levels in the human endometrium as determined via reverse transcription-quantitative PCR. (D) $O$-GlcNAcylation levels in human endometrial tissue as measured via western blotting at various phases of the menstrual cycle. Actin was used as a loading control. (E) Densitometry of $O$-GlcNAcylation protein levels normalized to actin. Data are presented as the mean \pm standard deviation of at least three independent experiments. ${ }^{* * *} \mathrm{P}<0.001$. $O$-GlcNAc, $O$-linked $\beta$ - $N$-acetylglucosamine; OGT, $O$-GlcNAc transferase.

\section{Results}

Expression of O-GlcNAc in the implantation-phase endometrium is higher than that of the non-implanted endometrium. The present study detected the expression of $O$-GlcNAcylation via immunohistochemistry in female endometrial tissue of the proliferative and secretory phase. As presented in Fig. 1, the results revealed that compared with the control (Fig. 1A-c and -f), levels of $O$-GlcNAc protein modification in the human secretory endometrium was higher than that of the endometrium in the proliferative phase, which was particularly low (Fig. 1A-a,-b,-d and-e). Additionally, $O$-GlcNAc-modified proteins were minimally expressed in the endometrium during the proliferative phase (Fig. 1A-a and -b). However, during the secretory phase, the expression of $O$-GlcNAc was significantly increased (Fig. 1A-d and -e, D and E). In humans, $O$-GlcNAc-modified proteins are mainly expressed in glandular epithelia and luminal epithelia (Fig. 1A-b and -e). Image analysis revealed that the mean optical density (OD) of $O$-GlcNAc in the secretory phase was significantly different compared with that of the proliferative phase $(\mathrm{P}<0.05$; Fig. 1B; Table I). Furthermore, the level of OGT mRNA in the secretory endometrium was significantly higher than that in the proliferative phase endometrium (Fig. 1C). 

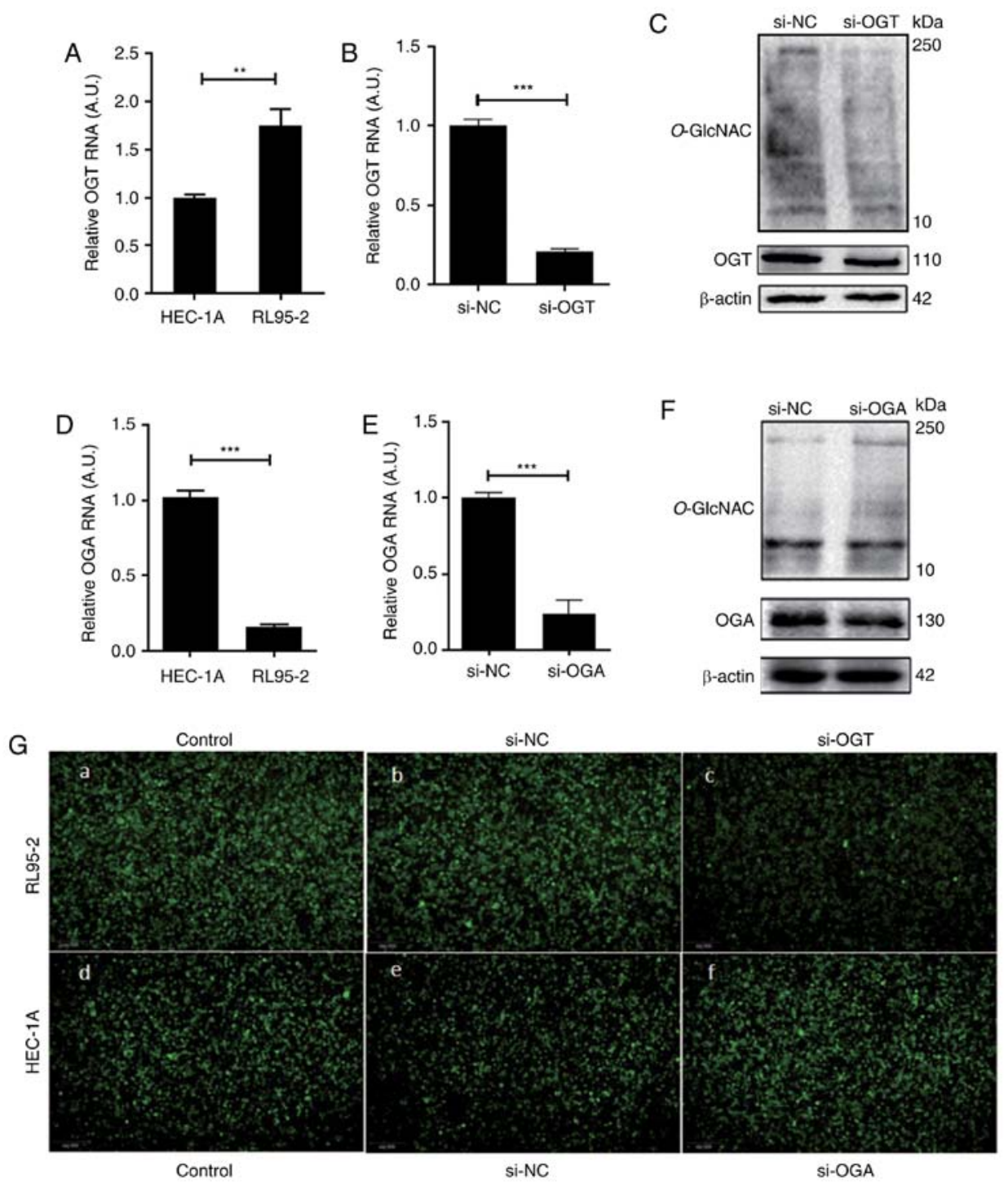

Figure 2. Influence of $O$-GlcNAcylation on the adhesion of JAR cells. (A) The expression of OGT in the RL95-2 cells and the HEC-1A cells via RT-qPCR (B) RT-qPCR analysis of OGT mRNA expression $48 \mathrm{~h}$ after transfection of si-OGT. (C) Western blotting revealed reduced protein expression of OGT and increased $O$-GlcNAcylation in RL95-2 cells that were treated with si-OGT. (D) The expression of OGA in the RL95-2 cells and the HEC-1A cells via RT-qPCR. (E) RT-qPCR analysis detecting OGA mRNA expression $48 \mathrm{~h}$ after transfection of si-OGA. (F) Western blotting results revealed reduced protein expressions of OGA and decreased $O$-GlcNAcylation in HEC-1A cells that were treated with si-OGA. (G) The adhesion of JAR cells to RL95-2 cells with control, si-NC and si-OGT (a-c). The adhesion of JAR cells to HEC-1A cells with control, si-NC and si-OGA (d-f). $O$-GlcNAc, $O$-linked $\beta$-N-acetylglucosamine; OGA, $O$-GlcNAcase; RT-qPCR, reverse transcription-quantitative PCR; siRNA, small interfering RNA; OGT, $O$-GlcNAc transferase. ${ }^{* *} \mathrm{P}<0.01,{ }^{* * * *} \mathrm{P}<0.001$.

Effect of O-GlcNAcylation on endometrial cell adhesion. The expression of OGT and OGA genes were detected via RT-qPCR in high receptive RL95-2 endometrial cells and low receptive HEC-1A endometrial cells. The results revealed that the expression of OGT in the RL95-2 cells was significantly higher than that in the HEC-1A cells (Fig. 2A). Conversely, the expression of OGA in the RL95-2 cells was significantly lower than that in the HEC-1A cells (Fig. 2D). In RL95-2 cells with a high OGT expression, an OGT siRNA was transfected to reduce the levels of $O$-GlcNAcylation, which was subsequently detected via RT-qPCR and western blotting. The results demonstrated that the level of OGT mRNA (Fig. 2B), OGT protein (Fig. 2C) and $O$-GlcNAcylation (Fig. 2C) were significantly decreased $48 \mathrm{~h}$ after transfection. However, following treatment with OGA siRNA in HEC-1A cells, the increased expression of OGA mRNA (Fig. 2E), OGA protein (Fig. 2F) and $O$-GlcNAc-modified proteins (Fig. 2F) was attenuated.
The effect of $O$-GlcNAcylation on the adhesion of endometrial cells to embryonic cells was studied by performing an adhesion assay. The results revealed that the adhesion of JAR cells to RL95-2 cells was stronger than that of HEC-1A cells (Fig. 2G-a and -d). Furthermore, the adhesion of JAR cells to RL-952 cells was significantly decreased following OGT siRNA transfection (Fig. 2G-b and -c). In addition, in HEC-1A cells, the adhesion of JAR cells to HEC-1A cells was significantly increased following transfection with OGA siRNA (Fig. 2G-e and -f).

Effect of O-GlcNAcylation on the proliferation of endometrial cells. To assess the role of $O$-GlcNAcylation in endometrial cells, OGA expression in HEC-1A cells and OGT expression in RL95-2 cells were experimentally reduced. The proliferation of cells was detected by performing a CCK- 8 assay. The results revealed that compared with the si-negative control 

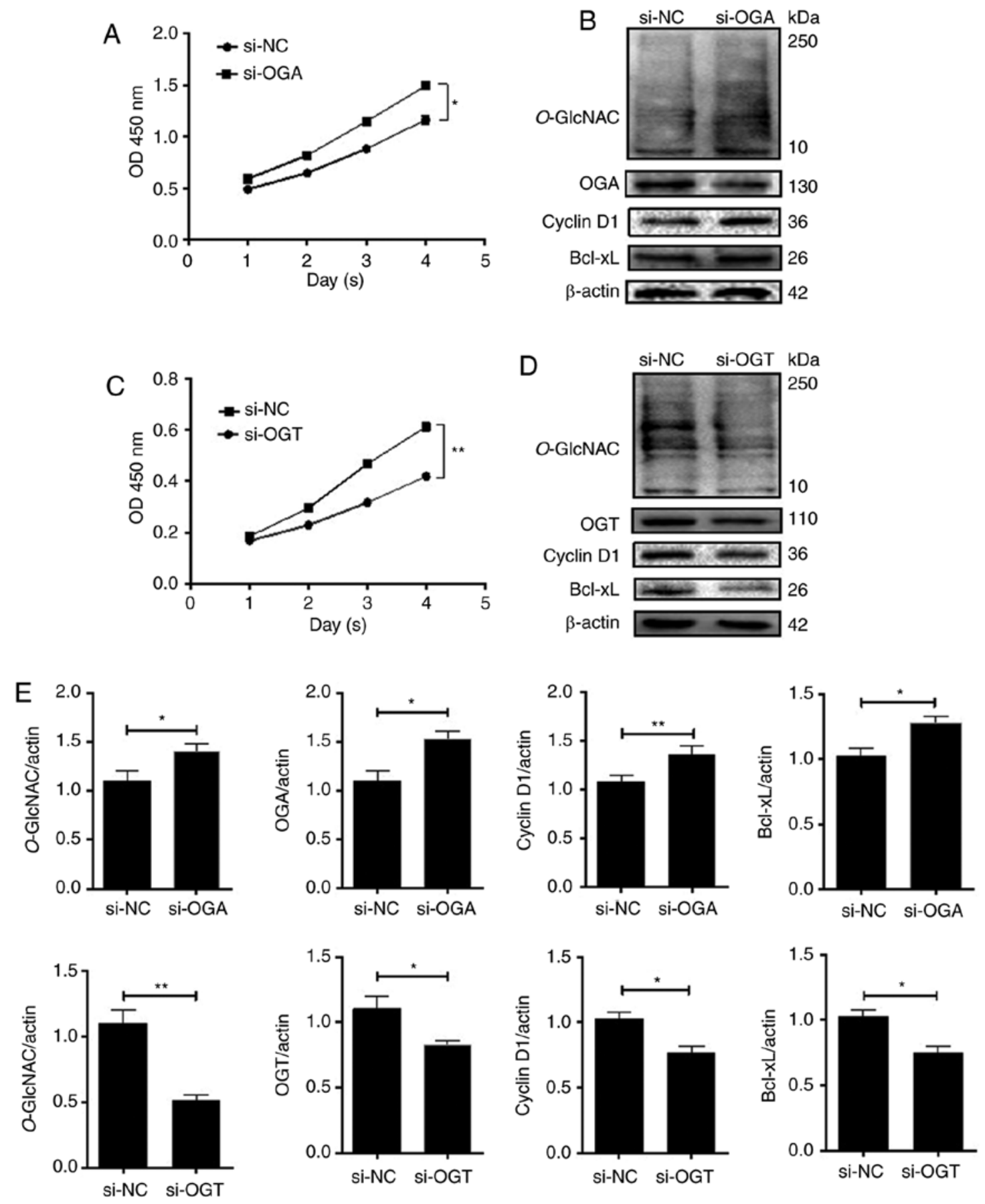

Figure 3. $O$-GlcNAc expression influences the proliferation of endometrial cells. (A) Treatment of HEC-1A cells with OGA-specific siRNA promoted cell proliferation. (B and E) si-OGA increased the expression of cyclin D1 and Bcl-xL. (C) Treatment of RL95-2 cells with OGT-specific siRNA reduced cell proliferation. (D and E) si-OGT decreased the expression of cyclin D1 and Bcl-xL. Data are presented as the mean \pm standard deviation of at least three independent experiments. ${ }^{*} \mathrm{P}<0.05$ and ${ }^{* *} \mathrm{P}<0.01$. $O$-GlcNAc, $O$-linked $\beta$ - $N$-acetylglucosamine; OGA, $O$-GlcNAcase; siRNA, small interfering RNA; Bcl-xL, B-cell lymphoma-xL; OGT, $O$-GlcNAc transferase.

Table I. Mean optical density value of $O$-GlcNAc in the endometrium of female tissue during different phases of the menstrual cycle.

Menstrual cycle phase

Mean optical density

Proliferative phase

$0.1088 \pm 0.024$

Secretory phase

$0.1837 \pm 0.017^{\mathrm{a}}$

${ }^{\mathrm{a}} \mathrm{P}<0.001$, significant difference.
(NC) group, the OD value of the HEC-1A si-OGA group was significantly increased over time. However, the OD value of the si-OGT group was significantly decreased over time in the RL95-2 cells. These results indicated that $O$-GlcNAcylation may enhance cellular proliferation (Fig. 3A and C). Western blotting was performed to assess the expression of the cellular proliferation-associated proteins cyclin D1 and Bcl-xL. After treatment with si-OGA to overexpress $O$-GlcNAc, levels of cyclin D1 and Bcl-xL in HEC-1A cells were significantly increased compared with the controls $(\mathrm{P}<0.01$ and $\mathrm{P}<0.05$; 
A

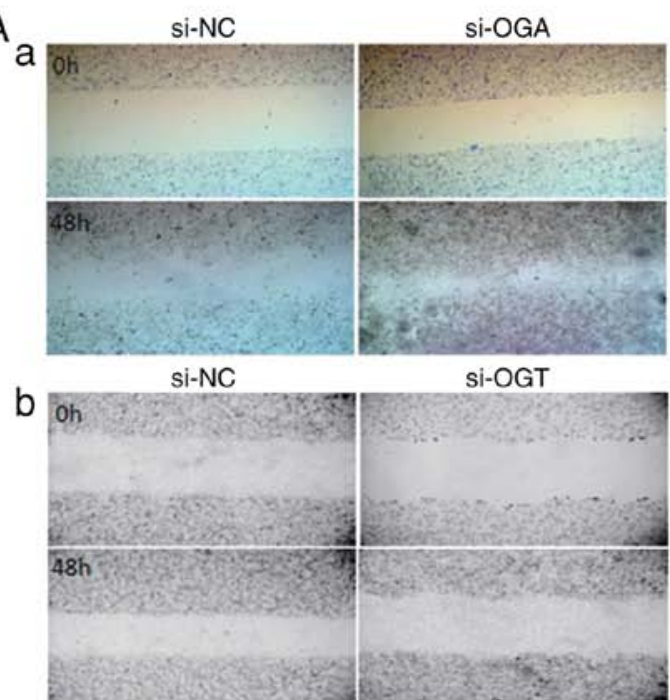

C a

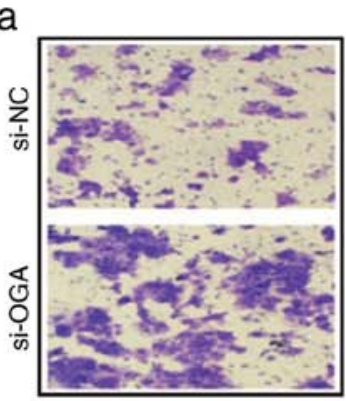

b

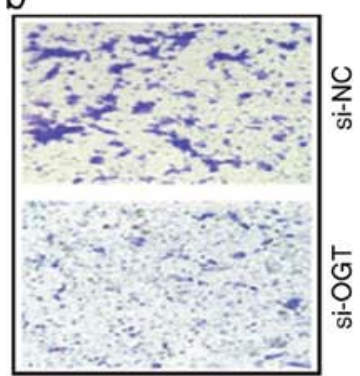

B

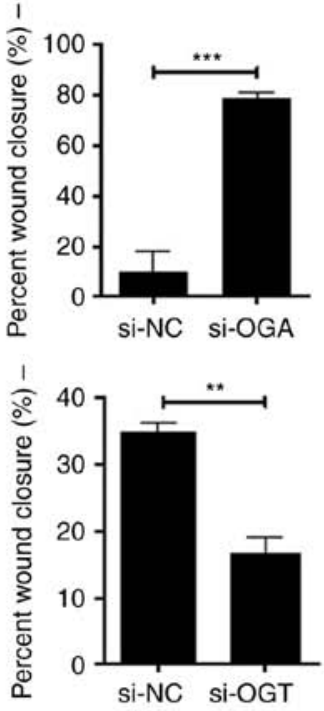

E
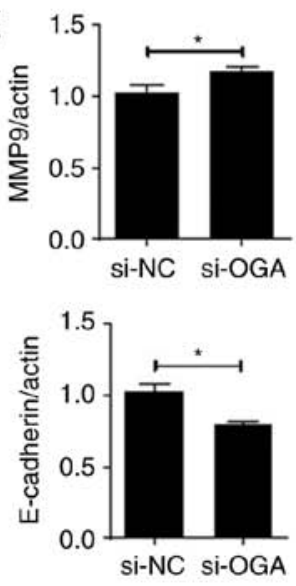
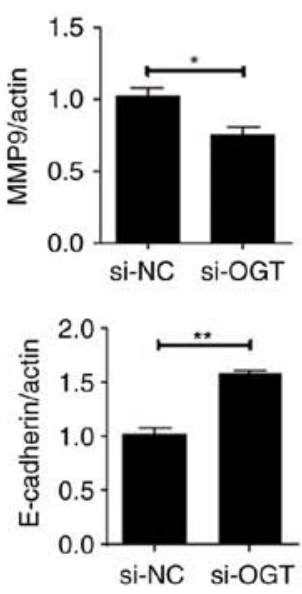
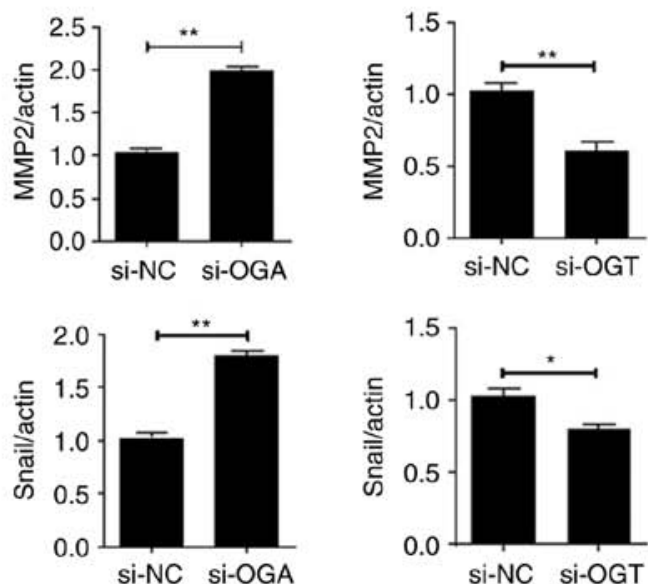

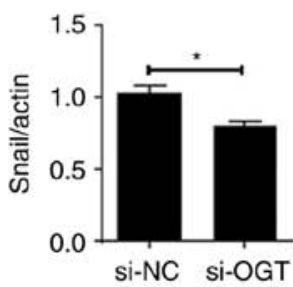

Figure 4. O-GlcNAc expression influences the migration and invasion of endometrial cells. (A-a, B and C-a) Treatment of HEC-1A cells with OGA-specific siRNA promoted cell migration and invasion. (D and E) si-OGA increased the expression of MMP9, MMP2 and Snail, and decreased the expression of E-cadherin. (A-b, B and C-b) Treatment of RL95-2 cells with si-OGT abolished cell migration and invasion. (D and E) si-OGT decreased the expression of MMP9, MMP2 and Snail, and increased the expression of E-cadherin. Data are presented as the mean \pm standard deviations of at least three independent experiments; ${ }^{*} \mathrm{P}<0.05,{ }^{* *} \mathrm{P}<0.01$ and ${ }^{* * *} \mathrm{P}<0.001$. $O$-GlcNAc, $O$-linked $\beta$ - $N$-acetylglucosamine; OGA, $O$-GlcNAcase; siRNA, small interfering RNA; MMP, matrix metallopeptidase; OGT, $O$-GlcNAc transferase.

Fig. 3B and E). The opposite was observed in RL-952 cells $(\mathrm{P}<0.05$; Fig. 3D and $\mathrm{E})$.

Effect of O-GlcNAcylation on the migration and invasion of endometrial cells. The migration and invasion of cells was detected via wound healing and Transwell invasion assays, respectively. Among HEC-1A cells, the OGA-siRNA group demonstrated increased migration (Fig. 4A-a and B) and invasion (Fig. 4C-a) abilities when compared with the si-NC group. Snail is the first member of the Snail family of proteins and it serves an important role in embryo implantation (14). During development, Snail, together with MMP family members, regulate a variety of extracellular matrix proteins by inhibiting the expression of E-cadherin (15). A key protein involved in cell cycle regulation is cyclin D1, which regulates the transition from $\mathrm{G} 1$ to $\mathrm{S}$ phase (16). Bcl-xL is an important member of the Bcl-2 family of proteins, that is an important anti-apoptotic protein (17). Western blot analysis demonstrated that levels of Snail were increased 
in the si-OGA group and that E-cadherin expression was negatively correlated with Snail expression ( $\mathrm{P}<0.05$; Fig. 4D and E). MMP2 and MMP9 expression were also significantly increased in the si-OGA group ( $\mathrm{P}<0.05$; Fig. $4 \mathrm{D}$ and $\mathrm{E})$. Furthermore, the OGT-siRNA group demonstrated reduced migration (Fig. 4A-b and B) and invasion (Fig. 4C-b) abilities of RL95-2 cells compared with the si-NC group. The expression levels of Snail, E-cadherin, MMP2 and MMP9 were opposite to those of the HEC-1A cells $(\mathrm{P}<0.05$; Fig. 4D and $\mathrm{E})$. The results indicated that $O$-GlcNAcylation may enhance cell migration and invasion by increasing the expression of related proteins.

\section{Discussion}

'Classical' protein glycosylation occurs only in cell membrane and secretory proteins. Glycosylation primarily consists of highly complex arrays of glycans, which can be divided into two types: $N$ - and $O$-glycans (18). The modification of proteins by $O$-GlcNAc ( $O$-GlcNAcylation) occurs in a myriad of carbohydrate post-translational modifications. Unlike 'traditional glycosylation', $O$-GlcNAc is generally not subject to further modifications that produce more complex glycans and is localized mainly within the cytoplasm or nucleoplasm (19).

Although the majority of glucose is metabolized via glycolytic pathways, $\sim 2-5 \%$ of the total glucose entering the cell is channeled into the nutrient-sensing hexosamine biosynthetic pathway (HBP) (20). In this pathway, the first and rate-limiting enzyme, glutamine: Fructose-6-phosphate amidotransferase, irreversibly uses an amino group from glutamine to convert fructose-6-phosphate into glucosamine-6-phosphate and glutamate. Glucosamine-6-phosphate is further metabolized to UDP-GlcNAc, which serves as the monosaccharide donor for $O$-GlcNAcylation (20). The level of $O$-GlcNAc modification in a protein is dependent on the concentration of the nucleotide sugar donor UDP-GlcNAc, a high energy compound second only to ATP (21). A common feature of cancer cells is the ability to increase glucose intake via the HBP pathway and elevate substrate UDP-GlcNAc levels, resulting in hyper- $O$-GlcNAcylation.

In recent years, there has been increasing evidence that $O$-GlcNAcylation and its regulators (OGT and OGA) serve an important role in the regulation of metabolic reprogramming in tumors by changing key transcription factors, metabolic enzymes and major carcinogenic signaling pathways (22-24). Two studies on breast and thyroid cancers revealed that the activity of OGA enzymes was increased in cancer tissue, although the expression levels of OGA protein were not determined $(9,25)$. In this study, western blotting results determined that the level of $O$-GlcNAcylation was decreased in certain tumor tissues compared with the control group. However, previous studies have demonstrated increased levels of $O$-GlcNAcylation in various cancer tissues. For example, compared with the corresponding para-cancerous tissue, $O$-GlcNAc modification was reported to be increased in breast, lung and colon cancer tissue sections $(26,27)$. The expression of OGT and OGA also appeared to increase in lung and colon tissues. Similarly, in patients with chronic lymphoblastic leukemia, the level of $O$-GlcNAc-modified proteins increased alongside OGT and OGA protein levels, when compared with normal lymphocytes (28). Therefore, $O$-GlcNAc modification may be a regulatory mechanism associated with metabolic changes and the pathogenesis of cancer.

Previous experimental results have revealed that $O$-GlcNAc modification is crucial for embryonic development. Knockout of the $O G T$ mouse gene exerts fatal effects on embryonic development (29). Jang et al (30) reported that octamer-binding transcription factor 4 and sex determining region-box 2 in embryonic stem cells are also glycosylated by $O$-GlcNAc and the absence of $O$-GlcNAcylation reduces the self-renewal ability of stem cells and hinders the reprogramming of somatic cells.

The human endometrium undergoes a complex series of organized proliferative and secretory changes in each menstrual cycle, exhibiting only a short period of receptivity, known as the 'window' for embryo implantation. Embryo implantation is the main limiting step during pregnancy and endometrial receptivity is closely associated with embryo implantation. The establishment of endometrial receptivity is important to ensure for the development of the fetus and placenta. The rate of embryo implantation and clinical pregnancy can be affected by the degree of endometrium receptivity.

The process of malignant tumor invasion and metastasis may be considered similar to that of embryo implantation. It has been reported that the epithelial to mesenchymal transition (EMT) process, which is important for tumor cell invasion $(31,32)$, is also necessary for embryo adhesion and invasion as it mediates the remodeling process of human endometrial cells (33). Therefore, the present study hypothesized that $O$-GlcNAcylation could also serve an important regulatory role in the establishment of endometrial receptivity and embryo implantation. To test this hypothesis, different clinical and hormonal tissue samples of human endometrium were collected. The tissue revealed a reduced expression of $O$-GlcNAc-modified proteins in the endometrium during the proliferative phase. However, $O$-GlcNAc levels increased significantly during the secretory phase. In addition, the expression of $O$-GlcNAcylation in glandular epithelia or luminal epithelia was higher than that of stromal cells.

The present study then utilized an in vitro implantation model to clarify the role and molecular mechanism of $O$-GlcNAc modification in endometrial receptivity. RNA interference was used to decrease or increase the expression of $O$-GlcNAcylation. OGT-siRNA was transfected into RL95-2 cells, and OGA-siRNA was transfected into HEC-1A cells. Cellular function assays were subsequently performed to detect the effects of $O$-GlcNAcylation on the proliferation, invasion and migration of endometrial cells. CCK-8 and wound healing assays were also performed to analyze cell proliferation and invasion and migration, respectively. Furthermore, the expression of EMT-associated proteins in RL95-2 and HEC-1A cells was detected. The results revealed that increased levels of $O$-GlcNAc modification promoted cell proliferation, migration and invasion, while also increasing the rate of cellular adhesion, thereby promoting embryo implantation and development. This indicated that during the embryo implantation, $O$-GlcNAc modification may promote the transformation of endometrial cell EMT and increase the migration of endometrial epithelial cells. The migration of epithelial cells causes the destruction and remodeling of the epithelial barrier at the implantation site, which changes the receptivity of the endometrium to promote embryo adhesion and implantation. 
However, the mechanisms involved in these processes are yet to be fully elucidated and require further study.

Over the past decade, significant progress has been made in our understanding of the broad role of post-translational modifications in basic cellular processes, including phosphorylation, acetylation, methylation and ubiquitination $(34,35)$. Although $O$-GlcNAc modification was discovered 30 years ago, its biological functions, including the modification of protein structures and interactions, cellular signaling, gene regulation, and physiological and metabolic regulation, have only begun to be understood. There is a growing body of evidence which indicates that $O$-GlcNAc serves as a nutritional sensor that links the metabolic state of a system with the regulation of cellular signal transduction, transcription and protein degradation (36). With respect to endometrial receptivity and embryo implantation, the precise role of $O$-GlcNAc modification in these complex processes still requires further elucidation.

\section{Acknowledgements}

Not applicable.

\section{Funding}

The present study was supported by the National Natural Scientific Grants (grant. nos. 31570798, 31971209, and 81901511), the Program for Liaoning Excellent Talents in University (grant no. LR2017042), by the Liaoning Key R\&D Program (2019JH2/10300017), and by the program for Liaoning Provincial Program for Top Discipline of Basic Medical Sciences.

\section{Availability of data and materials}

The datasets used during the present study are available from the corresponding author upon reasonable request.

\section{Authors' contributions}

YK and LS designed the experiments. XH, HL and XL performed the experiments. MD and $\mathrm{HZ}$ collected and analyzed the data. BY collected and analyzed the endometrial tissues. YX an AL interpreted the data for the study. All authors reviewed and approved the final version of the manuscript.

\section{Ethics approval and consent to participate}

Endometrial tissues were obtained from The First Affiliated Hospital of Dalian Medical University under the Human Research Agreement approved by the Ethics Committee of Dalian Medical University. Written informed consent was acquired from each patient prior to tissue collection.

\section{Patient consent for publication}

Not applicable.

\section{Competing interests}

The authors declare that they have no competing interests.

\section{References}

1. Torres CR and Hart GW: Topography and polypeptide distribution of terminal $\mathrm{N}$-acetylglucosamine residues on the surfaces of intact lymphocytes. Evidence for O-linked GlcNAc. J Biol Chem 259: 3308-3317, 1984.

2. Holt GD and Hart GW: The subcellular distribution of terminal $\mathrm{N}$-acetylglucosamine moieties. Localization of a novel protein-saccharide linkage, O-linked GlcNAc. J Biol Chem 261: 8049-8057, 1986.

3. Paria BC, Huet-Hudson YM and Dey SK: Blastocyst's state of activity determines the 'window' of implantation in the receptive mouse uterus. Proc Natl Acad Sci USA 90: 10159-10162, 1993.

4. Yoshinaga K: Uterine receptivity for blastocyst implantation. Ann N Y Acad Sci 541: 424-431, 1988.

5. Psychoyos A: Hormonal control of ovoimplantation. Vitam Horm 31: 201-256, 1973.

6. Lim HJ and Dey SK: HB-EGF: A unique mediator of embryo-uterine interactions during implantation. Exp Cell Res 315: 619-626, 2009.

7. Gu J, Fan J, Xu Y, Xie Y, Gong T and Kong Y: Regulatory function of $\beta 1,4$-galac tosyltransferase I expression on Lewis-Y glycan and embryo implantation. Gene 562: 220-225, 2015.

8. Gu J, Sui LL, Cui D, Ma YN, Zhu CY and Kong Y: Effects of LeY glycan expression on embryo implantation. Eur Rev Med Pharmacol Sci 20: 3327-3335, 2016.

9. Slawson C, Pidala J and Potter R: Increased N-acetyl-betaglucosaminidase activity in primary breast carcinomas corresponds to a decrease in $\mathrm{N}$-acetylglucosamine containing proteins. Biochim Biophys Acta 1537: 147-157, 2001.

10. Murray MJ and Lessey BA: Embryo implantation and tumor metastasis: Common pathways of invasion and angiogenesis. Semin Reprod Endocrinol 17: 275-290, 1999.

11. Hannan NJ, Paiva P, Dimitriadis E and Salamonsen LA: Models for study of human embryo implantation: Choice of cell lines? Biol Reprod 82: 235-245, 2010.

12. Schmittgen TD and Livak KJ: Analyzing real-time PCR data by the comparative C(T) method. Nat Protoc 3: 1101-1108, 2018.

13. Livak KJ and Schmittgen TD: Analysis of relative gene expression data using real-time quantitative PCR and the 2(-Delta Delta C(T)) method. Methods 25: 402-408, 2001.

14. Ma XH, Hu SJ, Yu H, Xu LB and Yang ZM: Differential expression of transcriptional repressor snail gene at implantation site in mouse uterus. Mol Reprod Dev 73: 133-141, 2006.

15. Dey SK, Lim H, Das SK, Reese J, Paria BC, Daikoku T and Wang H: Molecular cues to implantation. Endocr Rev 25: 341-373, 2004.

16. Baldin V, Lukas J, Marcote MJ, Pagano M and Draetta G: Cyclin D1 is a nuclear protein required for cell cycle progression in G1. Genes Dev 7: 812-821, 1993.

17. Yin XM: Signal transduction mediated by Bid, a pro-death Bcl-2 family proteins, connects the death receptor and mitochondria apoptosis pathways. Cell Res 10: 161-167, 2000.

18. Dennis JW, Nabi IR and Demetriou M: Metabolism, cell surface organization, and disease. Cell 139: 1229-1241, 2009.

19. de Queiroz RM, Carvalho E and Dias WB: O-GlcNAcylation: The sweet side of the cancer. Front Oncol 4: 132, 2014.

20. Marshall S, Bacote V and Traxinger RR: Discovery of a metabolic pathway mediating glucose-induced desensitization of the glucose transport system. Role of hexosamine biosynthesis in the induction of insulin resistance. J Biol Chem 266: 4706-4712, 1991.

21. Wells L, Vosseller K and Hart GW: A role for $\mathrm{N}$-acetylglucosamine as a nutrient sensor and mediator of insulin resistance. Cell Mol Life Sci 60: 222-228, 2003.

22. Lee TI and Young RA: Transcriptional regulation and its misregulation in disease. Cell 152: 1237-1251, 2013.

23. Koo CY, Muir KW and Lam EW: FOXM1: From cancer initiation to progression and treatment. Biochim Biophys Acta 1819: 28-37, 2012.

24. Khan Z and Bisen PS: Oncoapoptotic signaling and deregulated target genes in cancers: Special reference to oral cancer. Biochim Biophys Acta 1836: 123-145, 2013.

25. Krzeslak A, Pomorski L and Lipinska A: Elevation of nucleocytoplasmic beta- $\mathrm{N}$-acetylglucosaminidase (O-GlcNAcase) activity in thyroid cancers. Int J Mol Med 25: 643-648, 2010.

26. Gu Y, Mi W, Ge Y, Liu H, Fan Q, Han C, Yang J, Han F, Lu X and $\mathrm{Yu}$ W: GlcNAcylation plays an essential role in breast cancer metastasis. Cancer Res 70: 6344-6351, 2010. 
27. Mi W, Gu Y, Han C, Liu H, Fan Q, Zhang X, Cong Q and Yu W: $\mathrm{O}-$ GlcNAcylation is a novel regulator of lung and colon cancer malignancy. Biochim Biophys Acta 1812: 514-519, 2011.

28. Shi Y, Tomic J, Wen F, Shaha S, Bahlo A, Harrison R, Dennis JW, Williams R, Gross BJ, Walker S, et al: Aberrant O-GlcNAcylation characterizes chronic lymphocytic leukemia. Leukemia 24: 1588-1598, 2010.

29. Shafi R, Iyer SP, Ellies LG, O'Donnell N, Marek KW, Chui D, Hart GW and Marth JD: The O-GlcNAc transferase gene resides on the $\mathrm{X}$ chromosome and is essential for embryonic stem cell viability and mouse ontogeny. Proc Natl Acad Sci USA 97: 5735-5739, 2000

30. Jang H, Kim TW, Yoon S, Choi SY, Kang TW, Kim SY, Kwon YW, Cho EJ and Youn HD: O-GlcNAc regulates pluripotency and reprogramming by directly acting on core components of the pluripotency network. Cell Stem Cell 11: 62-74, 2012.

31. Lefebvre T, Pinte S, Guérardel C, Deltour S, Martin-Soudant N, Slomianny MC, Michalski JC and Leprince D: The tumor suppressor HIC1 (hypermethylated in cancer 1) is O-GlcNAc glycosylated. Eur J Biochem 271: 3843-3854, 2004.
32. Tai HC, Khidekel N, Ficarro SB, Peters EC and Hsieh-Wilson LC: Parallel identification of O-GlcNAc-modified proteins from cell lysates. J Am Chem Soc 126: 10500-10501, 2004.

33. Schlummer S, Vetter R, Kuder N, Henkel A, Chen YX, Li YM, Kuhlmann J and Waldmann H: Influence of serine $\mathrm{O}$-glycosylation or O-phosphorylation close to the vJun nuclear localisation sequence on nuclear import. Chembiochem 7: 88-97, 2006.

34. Teng CB, Diao HL, Ma XH, Xu LB and Yang ZM: Differential expression and activation of Stat 3 during mouse embryo implantation and decidualization. Mol Reprod Dev 69: 1-10, 2004

35. Cameron AM, Lawless SJ and Pearce EJ: Metabolism and acetylation in innate immune cell function and fate. Semin Immunol 28: 408-416, 2016.

36. Hart GW, Housley MP and Slawson C: Cycling of O-linked beta-N-acetylglucosamine on nucleocytoplasmic proteins. Nature 446: 1017-1022, 2007. 